\title{
Endoscopic neck dissection using peroral endoscopic myotomy for removal of extraluminal neck foreign body migrated out from the upper esophagus
}

A 50-year-old woman presented to the emergency room with a chief complaint of a foreign body sensation after eating steak prepared on a grill. A soft-tissue radiograph of the neck revealed a linear opacity at the level of the upper esophagus (C6-C7). A computed tomography (CT) scan of the neck was done, which showed a linear opacity between the upper esophagus and the trachea. An upper gastrointestinal endoscopy was performed, but revealed no foreign body present in the lumen of the esophagus. Under fluoroscopy guidance, the linear opacity was visualized at the level of C6 C7. An endoscopic ultrasound (EUS) was performed, as has been done in other cases for foreign body identification [1], and it revealed that the foreign body was present outside the muscularis propria of the esophagus. EUS-guided fineneedle aspiration (FNA) removal using a through-the-needle forceps was attempted, but was unsuccessful.

With the hope of identifying the foreign body for removal, a point of incision was selected using fluoroscopy to initiate a short peroral endoscopic myotomy (POEM) tunnel. Injections were performed to create a submucosal entry and, under multiplanar fluoroscopic guidance, the dissection was continued until we were in very close proximity to the foreign body. A transverse mucosal incision was initiated to increase the chances of identifying the object. The foreign body was identified in the deep muscularis propria of the esophagus protruding into the periesophageal space. The dissection was continued to free the foreign body. It was then retrieved intact using a rattoothed forceps and was identified as being a bristle from the grill brush. The area was then closed using through-thescope clips ( $\triangleright$ Video $\mathbf{1}$ ).

The patient had immediate resolution of her pain, which was constant prior to the procedure. She was admitted overnight for monitoring and a repeat soft-tissue

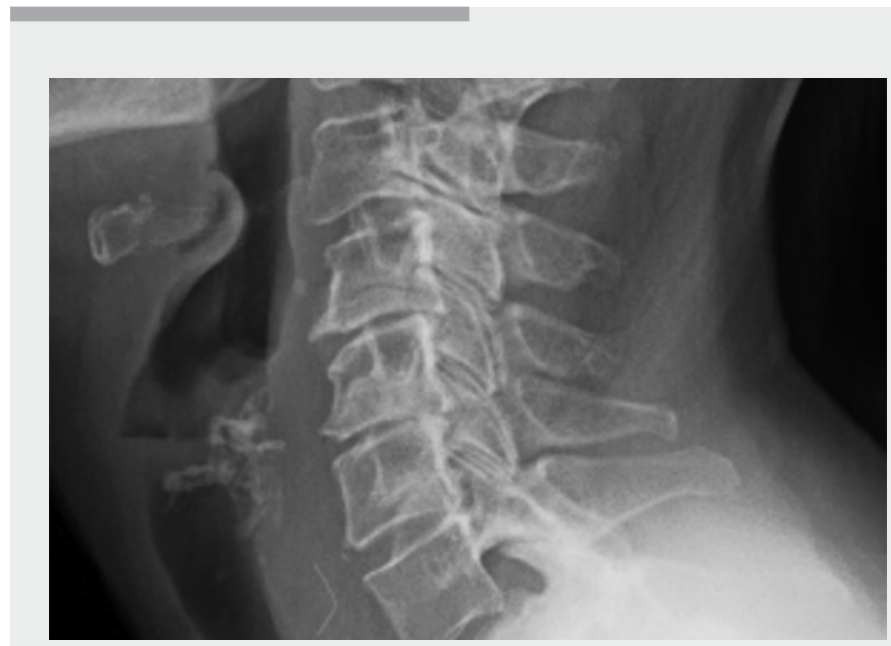

$\checkmark$ Video 1 Peroral endoscopic myotomy (POEM) technique is used to remove an extraluminal neck foreign body that had migrated outside the esophagus.

CT scan of the neck revealed resolution of the foreign body with mild tissue edema. She was discharged after 2 days with no complications.

Endoscopy_UCTN_Code_TTT_1AO_2AL

Competing interests

S. Andrawes is a consultant for Olympus America. A. Trindade is a consultant for Pentax Medical and Olympus America. P.C. Benias is a consultant for Medtronic, Boston Scientific, Apollo Endosurgical, and Olympus America. The remaining authors declare that they have no conflict of interest.

The authors

Sherif Andrawes ${ }^{1}$, Praneet Wander ${ }^{2}$, Adam Lackey ${ }^{1}$, Arvind J. Trindade ${ }^{2}$, Petros C. Benias ${ }^{2}$

1 Division of Gastroenterology, Staten Island University Hospital, Northwell Health, Staten Island, New York, USA

2 Division of Gastroenterology, Donald and Barbara Zucker School of Medicine at Hofstra/Northwell, Queens, New York, USA
Corresponding author

Petros C. Benias, MD

Long Island Jewish Medical Center, Division of Gastroenterology, Zucker School of Medicine at Hofstra/Northwell, Northwell Health System, 270-05 76th Avenue, New Hyde Park, NY 11040, USA

Fax: +1-718-470-5509

pbenias@northwell.edu

Reference

[1] Cao L, Chen N, Chen Y et al. Foreign body embedded in the lower esophageal wall located by endoscopic ultrasonography: a case report. Medicine 2018; 97: e11275

\section{Bibliography}

Endoscopy 2021; 53: E198

DOI 10.1055/a-1207-0231

ISSN 0013-726X

published online 2.9.2020

(C) 2020. Thieme. All rights reserved.

Georg Thieme Verlag KG; Rüdigerstraße 14,

70469 Stuttgart, Germany 